\title{
OBSERVATIONS OF BINARY STARS WITH THE DIFFERENTIAL SPECKLE SURVEY INSTRUMENT. IV. OBSERVATIONS OF KEPLER, CoRoT, AND HIPPARCOS STARS FROM THE GEMINI NORTH TELESCOPE
}

\author{
Elliott P. Horch ${ }^{1,5,6}$, Steve B. Howell ${ }^{2,5}$, Mark E. EveretT ${ }^{3,5}$, and David R. Ciardi ${ }^{4,5}$ \\ ${ }^{1}$ Department of Physics, Southern Connecticut State University, 501 Crescent Street, New Haven, CT 06515, USA; horche2@ southernct.edu \\ ${ }^{2}$ NASA Ames Research Center, Moffett Field, CA 94035, USA; steve.b.howell@ nasa.gov \\ ${ }^{3}$ National Optical Astronomy Observatory, 950 N. Cherry Ave, Tucson, AZ 85719, USA; everett@ noao.edu \\ ${ }^{4}$ NASA Exoplanet Science Institute, California Institute of Technology, 770 South Wilson Avenue, \\ Mail Code 100-22, Pasadena, CA 91125, USA; ciardi@ipac.caltech.edu \\ Received 2012 September 5; accepted 2012 October 2; published 2012 November 2
}

\begin{abstract}
We present the results of 71 speckle observations of binary and unresolved stars, most of which were observed with the DSSI speckle camera at the Gemini North Telescope in 2012 July. The main purpose of the run was to obtain diffraction-limited images of high-priority targets for the Kepler and CoRoT missions, but in addition, we observed a number of close binary stars where the resolution limit of Gemini was used to better determine orbital parameters and/or confirm results obtained at or below the diffraction limit of smaller telescopes. Five new binaries and one triple system were discovered, and first orbits are calculated for other two systems. Several systems are discussed in detail.
\end{abstract}

Key words: astrometry - binaries: visual - techniques: high angular resolution - techniques: interferometric techniques: photometric

\section{INTRODUCTION}

Since 2008, the Differential Speckle Survey Instrument (DSSI) has been used for two main projects at the WIYN $3.5 \mathrm{~m}$ Telescope ${ }^{7}$ at Kitt Peak: (1) to survey binary stars and suspected binaries discovered by Hipparcos and (2) to provide diffractionlimited imaging of targets of interest to the Kepler satellite mission. The instrument is described in Horch et al. (2009, hereafter Paper I) and subsequent WIYN observations have been discussed in Howell et al. (2011), as well as Horch et al. (2011a, 2011 b, hereafter Papers II and III, respectively). The last of these papers showed that, largely because of the fact that it records speckle observations in two colors simultaneously, DSSI can measure binary parameters below the diffraction limit at WIYN under certain conditions. The most important of these is that the source must be brighter than about the 10th magnitude, in order to ensure a high signal-to-noise ratio in the observation.

In 2012 July, we had the opportunity to use the instrument at the Gemini North Observatory on Mauna Kea, HI. With a primary mirror of $8.1 \mathrm{~m}$ in diameter, Gemini has an aperture size more than twice that of WIYN, so it offers the twin advantages of greater light gathering power and a smaller diffraction limit. The time awarded at Gemini was for diffraction-limited imaging of targets of the highest priority to Kepler-that is, those stars thought to harbor Earth-like planets based on Kepler transit data. DSSI speckle observations at Gemini are able to yield high signal-to-noise diffraction-limited images in two colors to greatly reduce the parameter space of false positives for planet

\footnotetext{
5 Visiting Astronomer, Gemini Observatory, National Optical Astronomy Observatory, which is operated by the Association of Universities for Research in Astronomy, Inc., under a cooperative agreement with the NSF on behalf of the Gemini partnership: the National Science Foundation (United States), the Science and Technology Facilities Council (United Kingdom), the National Research Council (Canada), CONICYT (Chile), the Australian Research Council (Australia), Ministério da Ciéncia, Tecnologia e Inovañao (Brazil), and Ministerio de Ciencia, Tecnología e Innovación Productiva (Argentina). 6 Adjunct Astronomer, Lowell Observatory.

7 The WIYN Observatory is a joint facility of the University of Wisconsin-Madison, Indiana University, Yale University, and the National Optical Astronomy Observatories.
}

detection (e.g., a background eclipsing binary blended with the target star). Ten hours of time were granted for this purpose. In addition, engineering time was given to us in evening twilight, when the telescope could not be used by other instruments in queue observing mode.

The installation of the camera went smoothly, so that we were able to use almost all of the engineering time to obtain science observations of known binary stars and other targets. These non-Kepler stars fulfilled four purposes: (1) two binaries with extremely well-known orbits were observed to help determine the scale and orientation of the pixel axes with respect to celestial coordinates; (2) a number of targets observed below the diffraction limit at WIYN were observed in order to confirm the WIYN results with a resolved observation at the larger aperture and/or provide data that would lead to an immediate orbit calculation; (3) four spectroscopic binaries thought to be thick disk members based on their metallicity and high proper motion were observed to add data for eventual calibration of a low-metallicity main-sequence mass-luminosity relation; and (4) two objects observed were high-priority targets for the CoRoT satellite mission. During the run, we also observed the Pluto-Charon system; these results are found in Howell et al. (2012).

In this paper, we detail the observations taken of the binary and unresolved stars, and characterize the astrometric and photometric performance of the DSSI at Gemini, insofar as that is possible given the small number of observations that we have. We also discovered six previously unknown systems (two from the Kepler target list, two bright stars that we used as point-source calibrators, and two others), and we determine orbital elements for two other systems. Based on the information at hand, we discuss the future value of these and certain other objects for stringent tests of our understanding of the mass-luminosity relation.

\section{OBSERVATIONS AND DATA REDUCTION}

The DSSI was installed on the telescope on 2012 July 25, and one first-light observation was obtained in twilight on that 
evening (UT July 26) after the pointing and focus were obtained. The observations described here were obtained on UT July 27 and 28, nominally comprising the first half of each night. Seeing on both nights was outstanding, usually between 0.4 and 0.5 arcsec.

For all of the observations discussed here, the filters used were a $692 \mathrm{~nm}$ center-wavelength filter with a $40 \mathrm{~nm}$ width in Channel A of the instrument, and an $880 \mathrm{~nm}$ center-wavelength filter of width $50 \mathrm{~nm}$ in Channel B. While these filters are somewhat wider than might typically be chosen for speckle observations at such a large telescope, we observed mainly at low airmass, where any residual atmospheric dispersion would be kept to a minimum. The filter width therefore allowed us to push the limiting magnitude as faint as possible, which was especially important for the Kepler science.

For brighter targets $(V<12)$, we typically took a sequence of 1000 speckle frames in each filter, where each frame was a $256 \times 256$ subarray on each chip centered on the target. Such a sequence consisted of $60 \mathrm{~ms}$ frames and required approximately 3 minutes to obtain. For fainter objects (and nearly all of the Kepler targets fell into this category), we took from three to eight such observations in sequence, depending on the faintness of the source. As has been our pattern at WIYN, we also periodically took point-source observations, to gauge dispersion and to have a high-quality speckle transfer function available to pair with each observation. This is needed to create the reconstructed images, as the target power spectrum must be divided by the speckle transfer function to yield the modulus of the object's Fourier transform.

\subsection{Pixel Scale and Orientation}

At WIYN, we have a custom-made slit mask which attaches to the telescope's tertiary mirror baffle support structure. This provides a precise scale measurement from first principles. At Gemini, using a slit mask was not feasible for so short a run, so we combined two methods to deduce our scale in this case. The first method was to focus on a reasonably bright star and offset the telescope in various directions from a nominal position by a few arcseconds (4, in the case of this run). In between each move, a $1 \mathrm{~s}$ exposure of the star was recorded. By computing centroids of each image, both scale and orientation can be derived. We have also used this method at WIYN, and found that while the orientation can be determined fairly precisely, the scale is less precise. Anticipating the same issue at Gemini, we observed two binaries with extremely well-known orbits (Grade 1 in the Sixth Orbit Catalog of Hartkopf et al. 2001a), computed their ephemeris positions based on the orbital elements, and derived both scale and orientation from these results.

The two binaries used were STF 1937AB (= HIP 75312) and HU 1176AB (= HIP 83838), which also have the benefit that the orientation in each case was essentially along one of the pixel axes on the detectors. This is important because, as we have written about in previous papers in this series, DSSI is known to have an optical effect in Channel B of the instrument that changes the pixel scale slightly as a function of position angle. We assumed that the same effect would be true at Gemini, but the two binaries gave us estimates of scale near the maximum and minimum values of the scale due to the distortion. Thus, we could approximately calibrate the effect for Gemini data using a minimum of observing time. In addition, we used the position angles obtained to make a slight correction to the orientation obtained from the $1 \mathrm{~s}$ images described above, since the position angle should be the same for a given binary star in both channels.
Since we have used these two objects in the determination of scale, we obviously do not report the relative astrometry for them in Section 3. However, we do report their relative photometry, which was not used in any calibration procedure.

\subsection{Reduction Method}

The analysis of the data is essentially the same as has been described in earlier papers in this series. We calculate the autocorrelation and triple correlation of each frame (see, e.g., Lohmann et al. 1983), and average these over the entire observation. In the Fourier domain, these functions are represented by the spatial frequency power spectrum and bispectrum, respectively. From these image products and a power spectrum of a point source, an estimate of the Fourier transform of the diffraction-limited image can be obtained using the method of Meng et al. (1990), and then filtered and inverse transformed to arrive at a reconstructed image. For a binary star observation, we typically use this image only to identify the secondary location (i.e., to make the quadrant determination) for use as the starting position for fitting the fringes observed in the power spectrum. However, in the case of unresolved objects, we do use the reconstructed images to estimate the detection limit as a function of separation from the primary star. This is discussed more fully in Section 3.4.

\section{RESULTS}

Our main results are presented in Tables 1 and 2. These include results on targets from all three satellite missions (Kepler, CoRoT, and Hipparcos). Table 1 contains the observations where the target was successfully resolved into two or more stars, and Table 2 contains high-quality non-detections. The columns in Table 1 are as follows: (1) the Washington Double Star (WDS) number (Mason et al. 2001b), which also gives the right ascension and declination for the object in 2000.0 coordinates; (2) the identifier from one of the following catalogs in the following priority order: (a) the Bright Star Catalogue (i.e., Harvard Revised [HR]) number, (b) Aitken Double Star (ADS) Catalogue number, (c) Henry Draper Catalogue (HD) number, (d) Durchmusterung (DM) number of the object, (e) GlieseJareiß Catalogue number, (f) Kepler Object of Interest (KOI) number, or (g) the Tycho identifier; (3) the Discoverer Designation; (4) the Hipparcos Catalogue number; (5) the Besselian date of the observation; (6) the position angle $(\theta)$ of the secondary star relative to the primary, with north through east defining the positive sense of $\theta ;(7)$ the separation of the two stars $(\rho)$ in arcseconds; (8) the magnitude difference $(\Delta m)$; (9) the center wavelength of the filter used in nm; and (10) the FWHM of the filter transmission in $\mathrm{nm}$. Position angles have not been precessed from the dates shown. Six objects in Table 1 have no previous detection in the 4th Catalogue of Interferometric Measures of Binary Stars (Hartkopf et al. 2001b); we propose discoverer designations of DSG (DSSI-Gemini) 1-6 here. Four observations come from the WIYN Telescope and have not previously been reported; this is for the specific intent of computing orbits for these objects in Section 4. In these cases, we have averaged the astrometry in both channels for higher precision, but we do not include these measures in the study below on Gemini measurement precision.

In Table 2, the column format is the following: (1) the celestial coordinates of the object, in the same format as Table $1 ;(2)$ the relevant identifier (KOI number or other); (3) the observation date, in Besselian year; $(4,5)$ the $5 \sigma$ detection limits in the $692 \mathrm{~nm}$ filter, as described in Section 3.4; and $(6,7)$ the $5 \sigma$ 
Table 1

Binary Star Speckle Measures

\begin{tabular}{|c|c|c|c|c|c|c|c|c|c|}
\hline $\begin{array}{l}\text { WDS } \\
(\alpha, \delta \mathbf{J} 2000.0)\end{array}$ & $\begin{array}{l}\text { HR,ADS } \\
\text { DM,etc. }\end{array}$ & $\begin{array}{l}\text { Discoverer } \\
\text { Designation }\end{array}$ & HIP & $\begin{array}{c}\text { Date } \\
\text { (Bess. Yr.) }\end{array}$ & $\begin{array}{c}\theta \\
\left({ }^{\circ}\right)\end{array}$ & $\begin{array}{c}\rho \\
(\prime \prime)\end{array}$ & $\Delta m$ & $\begin{array}{c}\lambda \\
(\mathrm{nm})\end{array}$ & $\begin{array}{c}\Delta \lambda \\
(\mathrm{nm})\end{array}$ \\
\hline \multirow[t]{2}{*}{$14404+2159$} & HR 5472 & MCA 40 & 71729 & 2012.5703 & 222.8 & 0.0309 & 1.14 & 692 & 40 \\
\hline & & & & 2012.5703 & 222.0 & 0.0309 & 1.16 & 880 & 50 \\
\hline \multirow[t]{2}{*}{$15232+3017$} & ADS 9617 & STF 1937AB & 75312 & 2012.5703 & $\ldots$ & $\ldots$ & 0.44 & 692 & $40^{\mathrm{a}}$ \\
\hline & & & & 2012.5703 & $\ldots$ & $\ldots$ & 0.39 & 880 & $50^{\mathrm{a}}$ \\
\hline \multirow[t]{2}{*}{$16546-0609$} & HR 6280 & DSG 1AB & 82730 & 2012.5730 & 272.0 & 0.3547 & 4.62 & 692 & 40 \\
\hline & & & & 2012.5730 & 271.0 & 0.3554 & 4.92 & 880 & 50 \\
\hline $16546-0609$ & HR 6280 & DSG 1AC & 82730 & 2012.5730 & 86.7 & 0.6553 & 6.34 & 880 & 50 \\
\hline \multirow[t]{2}{*}{$17080+3556$} & ADS 10360 & HU $1176 \mathrm{AB}$ & 83838 & 2012.5731 & $\ldots$ & $\ldots$ & 0.18 & 692 & $40^{\mathrm{a}}$ \\
\hline & & & & 2012.5731 & $\ldots$ & & 0.29 & 880 & $50^{\mathrm{a}}$ \\
\hline \multirow[t]{2}{*}{$17247+3802$} & BD+38 2932 & HSL $1 \mathrm{Aa}, \mathrm{Ab}$ & 85209 & 2012.5733 & 226.9 & 0.0269 & 0.36 & 692 & 40 \\
\hline & & & & 2012.5733 & 226.9 & 0.0271 & 0.38 & 880 & 50 \\
\hline \multirow[t]{2}{*}{$17247+3802$} & BD+38 2932 & HSL 1Aa,Ac & 85209 & 2012.5733 & 67.1 & 0.0944 & 1.99 & 692 & 40 \\
\hline & & & & 2012.5733 & 66.7 & 0.0951 & 1.74 & 880 & 50 \\
\hline \multirow[t]{2}{*}{$18084+4407$} & BD+44 2829 & HDS 2554 & 88852 & 2012.5705 & 21.0 & 0.1311 & 0.00 & 692 & $40^{\mathrm{b}}$ \\
\hline & & & & 2012.5705 & 21.1 & 0.1312 & 0.21 & 880 & $50^{\mathrm{b}}$ \\
\hline \multirow[t]{2}{*}{$18099+0307$} & ADS 11113 & YSC 132Aa,Ab & 89000 & 2012.5705 & 91.1 & 0.0144 & 0.00 & 692 & 40 \\
\hline & & & & 2012.5705 & 89.1 & 0.0138 & 0.00 & 880 & 50 \\
\hline \multirow[t]{2}{*}{$18439-0649$} & HR 7034 & YSC 133 & 91880 & 2012.5735 & 274.5 & 0.0790 & 0.61 & 692 & 40 \\
\hline & & & & 2012.5735 & 274.6 & 0.0788 & 0.62 & 880 & 50 \\
\hline \multirow[t]{2}{*}{$18582+7518$} & $\ldots$ & WOR 26 & 93119 & 2012.5707 & 154.6 & 0.2661 & 0.22 & 692 & 40 \\
\hline & & & & 2012.5707 & 154.7 & 0.2664 & 0.28 & 880 & 50 \\
\hline \multirow[t]{4}{*}{$19027+4307$} & $\mathrm{BD}+423233$ & YSC 13 & 93511 & 2012.0948 & 166.0 & 0.0905 & 0.93 & 692 & $40^{\mathrm{c}}$ \\
\hline & & & & 2012.0948 & $\ldots$ & $\ldots$ & 0.87 & 880 & $50^{\mathrm{c}}$ \\
\hline & & & & 2012.5707 & 174.5 & 0.0821 & 0.89 & 692 & 40 \\
\hline & & & & 2012.5707 & 174.6 & 0.0826 & 0.89 & 880 & 50 \\
\hline \multirow[t]{2}{*}{$19062+4926$} & KOI 1422 & DSG 2 & $\ldots$ & 2012.5707 & 216.5 & 0.2221 & 1.72 & 692 & 40 \\
\hline & & & & 2012.5707 & 215.9 & 0.2185 & 1.62 & 880 & $50^{\mathrm{d}}$ \\
\hline \multirow[t]{2}{*}{$19108+4720$} & KOI 98 & KOI 98AB & $\ldots$ & 2012.5708 & 144.4 & 0.2893 & 0.44 & 692 & 40 \\
\hline & & & & 2012.5708 & 144.5 & 0.2901 & 1.09 & 880 & 50 \\
\hline \multirow[t]{2}{*}{$19111+3847$} & ADS 12145 & STF $2481 \mathrm{~A}$ & 94252 & 2012.5708 & 32.9 & 0.0067 & 0.90 & 692 & 40 \\
\hline & & & & 2012.5708 & 30.3 & 0.0079 & 0.76 & 880 & 50 \\
\hline \multirow[t]{2}{*}{$19143+4239$} & TYC 3129-2599-1 & DSG 3 & $\ldots$ & 2012.5736 & 331.3 & 0.0288 & 0.00 & 692 & $40^{\mathrm{d}}$ \\
\hline & & & & 2012.5736 & 332.1 & 0.0288 & 0.26 & 880 & $50^{\mathrm{d}}$ \\
\hline \multirow[t]{2}{*}{$19190+3916$} & ADS 12310 & STF 2502AB & 94924 & 2012.5708 & 203.0 & 1.1507 & 2.25 & 692 & $40^{\mathrm{e}}$ \\
\hline & & & & 2012.5708 & 202.9 & 1.1517 & 1.72 & 880 & $50^{\mathrm{e}}$ \\
\hline $19262+3619$ & HR 7395 & DSG 4 & 95556 & 2012.5708 & 90.8 & 0.0189 & 0.00 & 692 & $40^{\mathrm{d}}$ \\
\hline & & & & 2012.5708 & 264.7 & 0.0185 & 0.16 & 880 & $50^{\mathrm{d}}$ \\
\hline & & & & 2012.5708 & 92.7 & 0.0191 & 0.00 & 692 & 40 \\
\hline & & & & 2012.5708 & 270.8 & 0.0196 & 0.30 & 880 & $50^{\mathrm{d}}$ \\
\hline $19264+4928$ & GJ 1237 & YSC 134 & 95575 & 2011.4511 & 34.7 & 0.0313 & 0.70 & 692 & $40^{\mathrm{c}}$ \\
\hline & & & & 2011.4511 & $\ldots$ & $\ldots$ & 0.78 & 880 & $50^{\mathrm{c}}$ \\
\hline & & & & 2012.5738 & 199.0 & 0.0285 & 0.76 & 692 & 40 \\
\hline & & & & 2012.5738 & 200.1 & 0.0283 & 0.59 & 880 & 50 \\
\hline $19376+4407$ & KOI 2474 & DSG 5 & $\ldots$ & 2012.5738 & 282.4 & 0.5797 & 0.87 & 692 & 40 \\
\hline & & & & 2012.5738 & 282.5 & 0.5771 & 0.94 & 880 & $50^{\mathrm{d}}$ \\
\hline $19380+3354$ & GJ 4118 & YSC 135 & 96576 & 2012.5711 & 2.1 & 0.0344 & 0.16 & 692 & $40^{\mathrm{d}}$ \\
\hline & & & & 2012.5711 & 1.9 & 0.0345 & 0.04 & 880 & $50^{\mathrm{d}}$ \\
\hline $19467+4421$ & BD+43 3359 & YSC 136 & 97321 & 2012.5738 & 129.2 & 0.0568 & 1.37 & 692 & 40 \\
\hline & & & & 2012.5738 & 129.5 & 0.0567 & 1.27 & 880 & 50 \\
\hline $20599+4016$ & HD 200077 & COU 2431Aa & 103641 & 2012.5738 & 257.8 & 0.0218 & 1.20 & 692 & 40 \\
\hline & & & & 2012.5738 & 256.7 & 0.0231 & 1.21 & 880 & $40^{\mathrm{d}}$ \\
\hline $21041+0300$ & HD 200580 & WSI 6AB & 103987 & 2012.5739 & 269.5 & 0.2265 & 1.83 & 692 & 40 \\
\hline & & & & 2012.5739 & 269.6 & 0.2322 & 1.71 & 880 & 50 \\
\hline $21041+0300$ & HD 200580 & DSG 6Aa & 103987 & 2012.5739 & 224.8 & 0.0149 & 1.51 & 692 & 40 \\
\hline & & & & 2012.5739 & 196.7 & 0.0151 & 1.89 & 880 & 50 \\
\hline
\end{tabular}

Notes.

a Astrometry for this observation does not appear because it was used in the determination of the scale.

b The quadrant determination here is inconsistent with previous measures appearing in the 4th Interferometric Catalog.

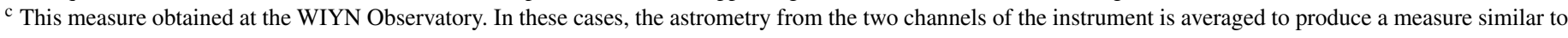
Gemini in precision.

${ }^{\mathrm{d}}$ Quadrant ambiguous.

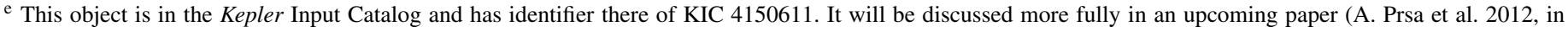
preparation). 

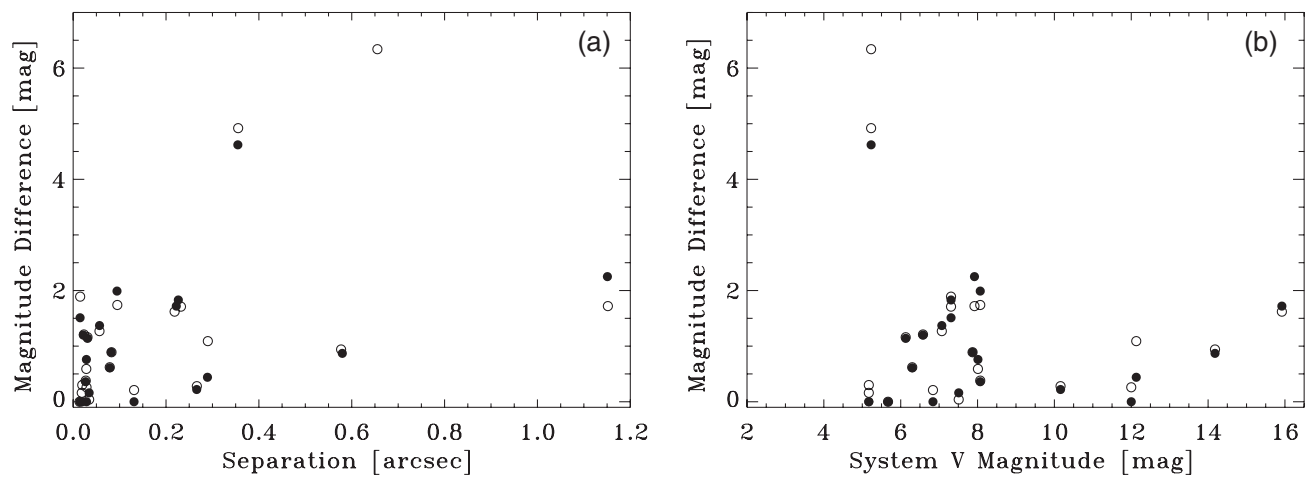

Figure 1. (a) Magnitude difference as a function of separation for the measures listed in Table 1. (b) Magnitude difference as a function of system $V$ magnitude for the measures listed in Table 1. In both plots, the open circles are measures taken with the $880 \mathrm{~nm}$ filter and filled circles are measures in the $692 \mathrm{~nm}$ filter. The four WIYN measures in Table 1 are not included here.

Table 2

High-quality Non-detections and $5 \sigma$ Detection Limits

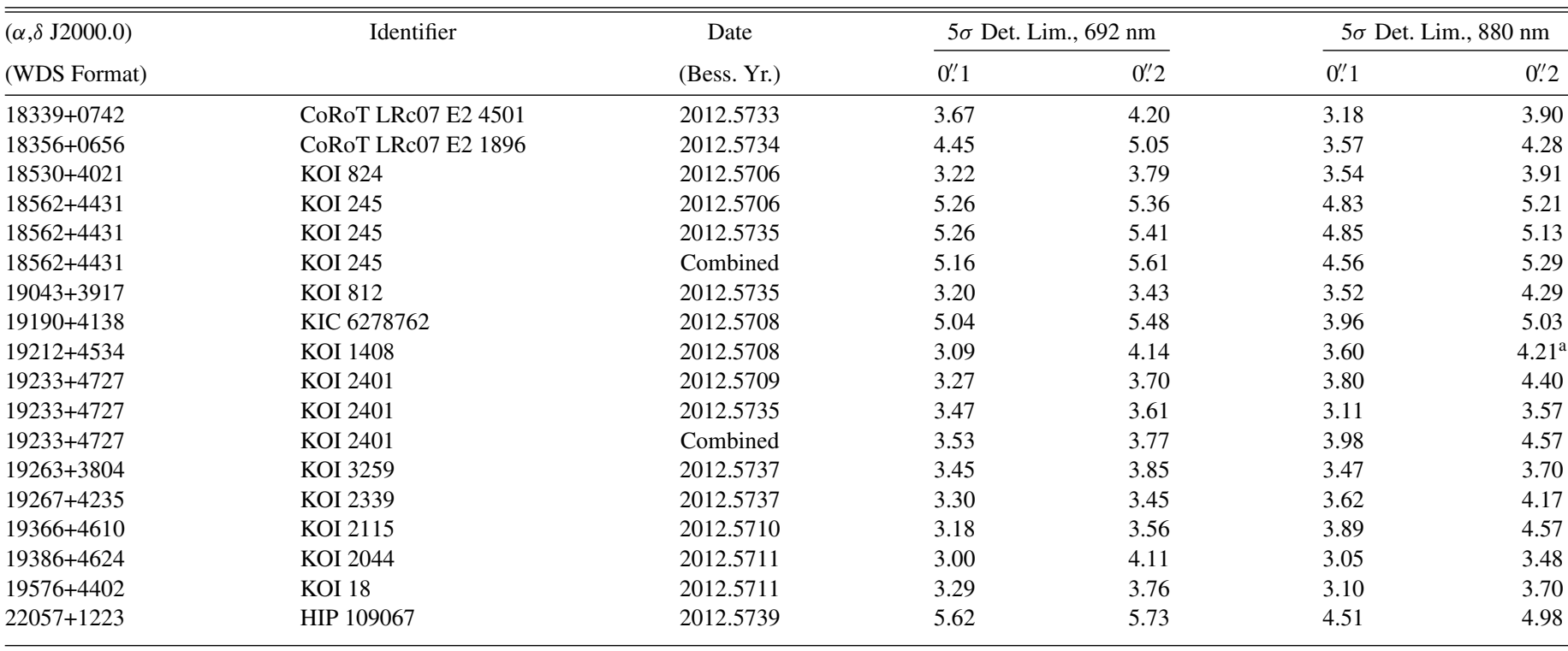

Note. ${ }^{\text {a }}$ There is some weak evidence for a small-separation $(<0$ '! 1$)$ companion to this object. While the limits here are valid for the separations listed, one should therefore view this object as a "suspected double."

detection limits in the $880 \mathrm{~nm}$ filter. Most of these objects are drawn from the Kepler list of high-priority targets, but one, namely HIP 109067, is a known single-lined spectroscopic binary with low metallicity, and two others are high-priority objects of interest for the CoRoT satellite mission.

\subsection{Detection Limits}

It is not possible with so few measures to conduct a detailed analysis of the detection limits of DSSI at Gemini. However, we can at least compare the results here with WIYN data and give a preliminary picture regarding the similarities and differences between the two apertures. To that end, we have constructed plots of observed magnitude difference as a function of both separation and target $V$ magnitude in Figures 1(a) and (b), respectively. These can be directly compared with Figure 1 in Paper II in this series. While sparse, the Gemini plots appear comparable at this stage to the WIYN plots, except that Gemini data extend to smaller separations to larger magnitude differences than at WIYN, as one would expect. The largest magnitude difference that we successfully measured was 6.34 for the wider component in the $880 \mathrm{~nm}$ observation of HR
6280 (= DSG 1 AC); this star was not detected in the $692 \mathrm{~nm}$ observation. Assuming that this object is a very red star on the main sequence, it would have a smaller magnitude difference at $880 \mathrm{~nm}$ than $692 \mathrm{~nm}$, and therefore it is likely that we do not have the dynamic range in a single 1000 frame observation to make the detection at the shorter wavelength even though it does appear in the $880 \mathrm{~nm}$ image.

While the faint end of Figure 1(b) does not look that different from the same figure in Paper II, this is almost certainly due to the small number of binaries observed in this region of the diagram. To examine this further, we compare in Figure 2 two reconstructed images of $\mathrm{KOI} 98 \mathrm{AB}$, a Kepler target that we discovered at WIYN as a double star and first reported in Howell et al. (2011). The first image reconstruction is one of the best images obtained at WIYN, in seeing of 0.7 arcsec. The second image is the reconstructed image obtained from the Gemini data. There is clear and significant difference in signal-to-noise ratio in favor of the Gemini data. Noise fluctuations in the WIYN image are approximately six times higher relative to the primary peak than in the Gemini image; in addition, the WIYN image is clearly lower in resolution. 

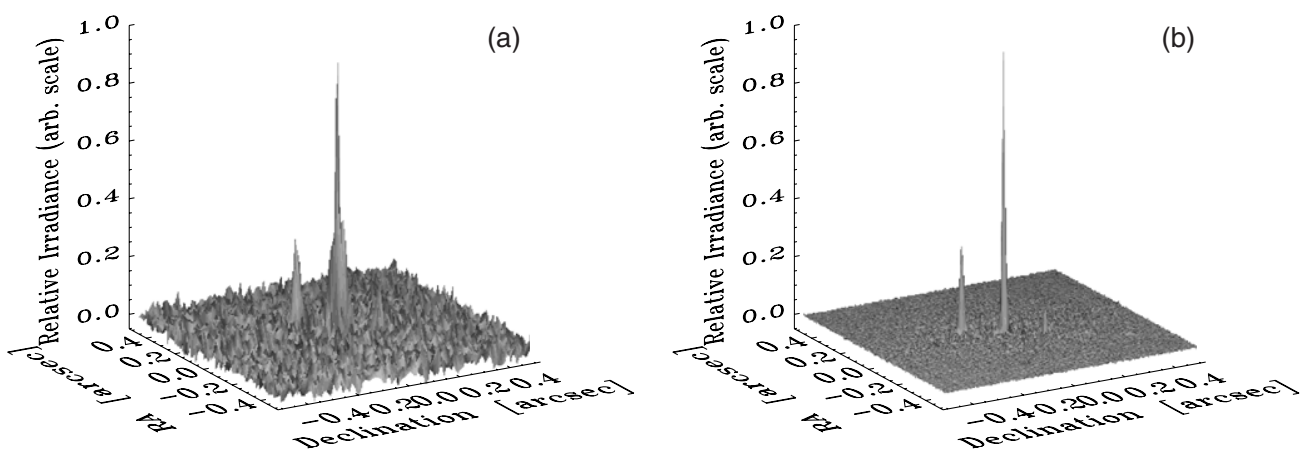

Figure 2. Reconstructed images of KOI 98AB, a 12th magnitude object, in the $692 \mathrm{~nm}$ filter. (a) The image obtained at the WIYN $3.5 \mathrm{~m}$ telescope, UT 2010 October 23. (b) The image obtained at Gemini, UT 2012 July 27.
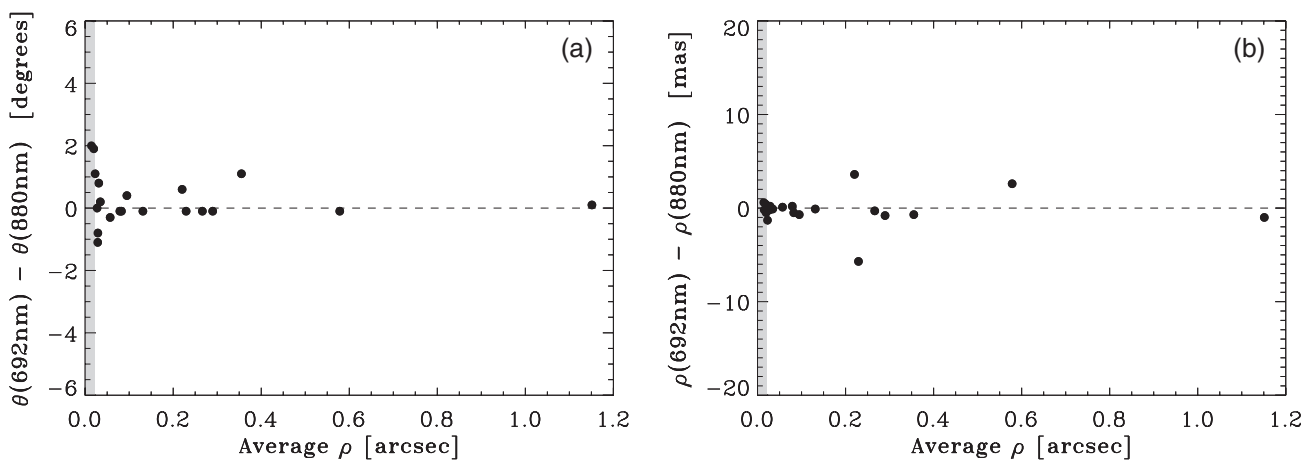

Figure 3. Measurement differences between the two channels of the instrument plotted as a function of measured separation, $\rho$. (a) Position angle $(\theta)$ differences. (b) Separation $(\rho)$ differences. In both plots, the gray band at the left marks the region below the diffraction limit of the telescope.

\subsection{Astrometric Accuracy and Precision}

To study the quality of the astrometry in the data set, we employed two methods. First, we compared the results in the two channels to determine the repeatability of the results. This is shown in Figure 3. We find that, for measures above the diffraction limit (nominally $0.02 \mathrm{arcsec}$ ), the average of the position angle differences is $0.08 \pm 0.13$. For the same group of measures, the average difference in separation is $-0.26 \pm 0.43$ mas. These values give good confidence that the method for scale determination in both channels gives consistent results. Larger differences in position angle occur for measures near and below the diffraction limit; this is expected as the linear measurement uncertainty subtends a larger angle at smaller separations. In the plot for separation (Figure 3(b)), there are three points that are farther from the zero line than the other points. Two of these three points are KOI 1422 and KOI 2474. Both are faint targets (16th and 14th magnitude, respectively), and so a loss of signal-to-noise ratio probably accounts for degraded astrometry here. The third point is HD 200580, which is a challenging triple system with a sub-diffractionlimited component and a third component at a separation of approximately 0.25 arcsec from the primary. It may be that some residual atmospheric dispersion, combined with the difficulty of fitting the interfering fringes in the Fourier plane, can account for a larger difference in separation obtained here.

The standard deviation of the position angle differences is $0.57 \pm 0.13$, and for separation, the result is $1.81 \pm 0.30$ mas. These numbers give some information about the level of repeatability (i.e., random uncertainty) in our measures. In this group of measures, the average separation is 0.206 arcsec. 1.83 mas would subtend an angle of 0.50 at that separation, so we can surmise that the two orthogonal coordinates of position angle and separation are completely consistent with regard to the repeatability between channels. The standard deviation of the differences between the channels will be $\sqrt{2}$ times larger than the uncertainties in each channel, assuming Gaussian statistics, so that a first estimate of the uncertainty in each measure listed in Table 1 (assuming systematic errors are not significant) is $1.81 / \sqrt{2}=1.3 \pm 0.2$ mas. If the astrometry from both channels is averaged together (which we did not do in Table 1), then a further reduction in uncertainty of $\sqrt{2}$ is obtained, meaning that DSSI appears to be capable of astrometry to at least the 0.9 mas level at Gemini in a typical 3 minute observation.

Besides the two binaries used in the scale calibration, three other objects in the list have orbits in the Sixth Orbit Catalog. These are MCA 40 (a Grade 2 orbit), HSL 1Aa,Ab (a Grade 3 orbit), and HDS 2554 (a Grade 4 orbit). We show the ephemeris predictions for the astrometry of these systems together with residuals obtained when comparing to the Gemini measures in Table 3. Given the small number of measures with which to make the comparison, as well as the sizable uncertainties in the orbital ephemerides, it is fruitless to make too much of these results. We would only note at this point that there is no evidence for systematic errors in the astrometry, and that the standard deviation of the separation residuals, which is 1.96 mas, is consistent with the repeatability number in this coordinate, despite some of the scatter certainly coming from the orbital uncertainties themselves.

\subsection{Photometric Accuracy and Precision}

As a first step toward understanding the precision of our photometric results, we studied the one object where we have 
Table 3

Comparison with Orbital Ephemerides

\begin{tabular}{|c|c|c|c|c|c|c|c|c|}
\hline WDS & $\begin{array}{l}\text { Discoverer } \\
\text { Designation }\end{array}$ & $\begin{array}{c}\text { Orbit } \\
\text { Reference }\end{array}$ & $\begin{array}{c}\theta_{\mathrm{obs}} \\
\left(^{\circ}\right)\end{array}$ & $\begin{array}{c}\rho_{\mathrm{obs}} \\
\text { (mas) }\end{array}$ & $\begin{array}{c}\theta_{\mathrm{eph}} \\
\left(^{\circ}\right)\end{array}$ & $\begin{array}{l}\rho_{\mathrm{eph}} \\
\text { (mas) }\end{array}$ & $\begin{array}{c}\theta \text { Res. }^{\mathrm{a}} \\
\left({ }^{\circ}\right)\end{array}$ & $\begin{array}{c}\rho \text { Res. }^{\mathrm{a}} \\
\text { (mas) }\end{array}$ \\
\hline $14404+2159$ & MCA 40 & Paper III & 222.8 & 30.9 & $211.7 \pm 34.3$ & $26.5 \pm 9.7$ & 11.1 & 4.4 \\
\hline $14404+2159$ & MCA 40 & Paper III & 222.0 & 30.9 & $211.7 \pm 34.3$ & $26.5 \pm 9.7$ & 10.3 & 4.4 \\
\hline $17247+3802$ & HSL $1 \mathrm{Aa}, \mathrm{Ab}$ & Horch et al. (2006) & 226.9 & 26.9 & $234.3 \pm 2.1$ & $29.7 \pm 1.8$ & -7.4 & 2.8 \\
\hline $17247+3802$ & HSL $1 \mathrm{Aa}, \mathrm{Ab}$ & Horch et al. (2006) & 226.9 & 27.1 & $234.3 \pm 2.1$ & $29.7 \pm 1.8$ & -7.4 & 2.6 \\
\hline $18084+4407$ & HDS 2554 & Paper III & 201.0 & 131.1 & $204.2 \pm 4.4$ & $131.1 \pm 11.1$ & $-3.2^{\mathrm{b}}$ & 0.0 \\
\hline $18084+4407$ & HDS 2554 & Paper III & 201.1 & 131.2 & $204.2 \pm 4.4$ & $131.1 \pm 11.1$ & $-3.1^{b}$ & 0.1 \\
\hline
\end{tabular}

Notes.

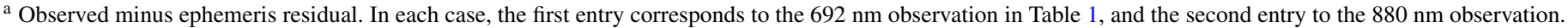

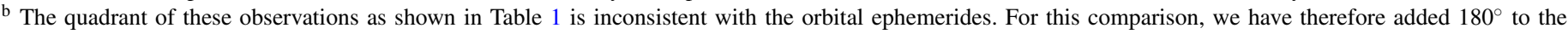
observed value prior to forming the residual.

multiple files, each of which yielded a usable binary result: KOI 2474. While only the summed result is shown in Table 1, we have eight individual observations of 1000 frames each, and can compute the standard deviation of the magnitude differences obtained to estimate internal precision. When doing this, we obtain $0.064 \pm 0.019 \mathrm{mag}$ in the $692 \mathrm{~nm}$ filter and $0.19 \pm 0.05 \mathrm{mag}$ in the $880 \mathrm{~nm}$ filter. This is a faint source ( $R \sim 14.2$ ), so these numbers should effectively be viewed as near the upper limit of the repeatability of the photometry.

When assessing the quality of relative photometry in our previous papers, we have used the magnitude differences appearing in the Hipparcos Catalogue. However, in doing so here, two problems are encountered: (1) only a few objects in Table 1 were resolved by Hipparcos due to the large difference in aperture size between the satellite's telescope and Gemini, and (2) there is no good comparison filter, as the Hipparcos filter is slightly bluer than $V$ and the shortest-wavelength filter used in our Gemini observations was $692 \mathrm{~nm}$. Therefore, the only meaningful comparison that can be made at this time is with other large-aperture speckle observations taken in the same filter or a similar filter. A number of such measures appear in the 4th Interferometric Catalog, and so we require that the filter wavelength listed there be within $20 \mathrm{~nm}$ of our $(692 \mathrm{~nm})$ filter, and that at least two measures be available from previous observations so that an estimate of the uncertainty can be found from the standard error of these measures. This leaves us with 11 systems, which we plot in Figure 4. (There are many fewer observations similar in wavelength to our $880 \mathrm{~nm}$ filter in the 4th Interferometric Catalog, so we did not pursue a comparison at that wavelength.) While preliminary, these data suggest that there is no evidence for a systematic offset of the Gemini results from previous photometry. In addition, if one considers the "best" quality objects, i.e., those with standard errors less than $0.1 \mathrm{mag}$ in the 4th Interferometric Catalog, and one forms a difference in $\Delta m$ between the previous data and the Gemini data, these have a standard deviation of approximately 0.1 mag. For this sample, the mean standard error of the previous photometry is $0.062 \mathrm{mag}$. So, to estimate the intrinsic scatter in the Gemini photometry, we can subtract this value in quadrature from 0.1 , whereupon we obtain $0.08 \mathrm{mag}$, comparable to the repeatability study of KOI 2474.

\subsection{Non-detections}

In Paper II, we described a system for estimating the detection limit for faint companions that was based on the reconstructed images that we obtain from the data. The method is to consider each local maximum in the reconstructed image as a potential

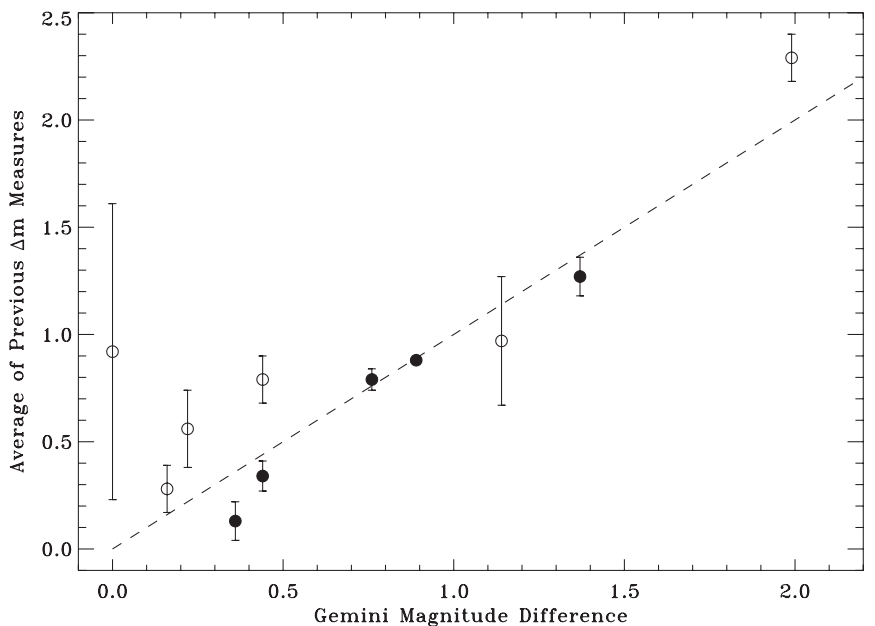

Figure 4. Comparison of the differential photometry presented in Table 1 with existing differential photometry. We plot the average value of the magnitude difference from values in the 4th Interferometric Catalog in comparable filters to $692 \mathrm{~nm}$ vs. the Gemini result. Open circles are plotted for systems with larger uncertainties given the data in the 4th Interferometric Catalog, filled circles indicate those systems with a $\sigma_{\Delta m}<0.1$ mag from the data in the 4th Interferometric Catalog, and the dashed line is drawn at $y=x$.

stellar source, and to determine the statistics of these peaks as a function of distance from the primary star. Specifically, the average value and standard deviation of all local maxima in annuli centered on the primary star is computed, and the detection limit is then chosen as the average value plus five times the standard deviation. In our previous work at WIYN, we saw that this $5 \sigma$ limit is a function of distance from the primary, with the limiting magnitude being fainter the farther the position is from the primary star. The statistics of local minima, which we have previously found to be comparable to those of the local maxima, are also used in the calculation of the final $5 \sigma$ detection limit.

In constructing Table 2, we have followed this method with one addition: for the WIYN work, we have reported $5 \sigma$ limits at a distance of 0.2 arcsec from the primary; at Gemini, the increased resolution permits us to move closer to the primary, so that in Table 2 we have reported the limiting magnitude at both 0.1 and 0.2 arcsec. In Figure 5, we show examples of the calculation, for one non-detection (our "combined" observation of KOI 245 as described below) and one discovery detection (the triple system HR 6280 = DSG 1). In these plots, we calculate and show the $5 \sigma$ detection limit as a function of separation from the object of interest. This is drawn as a dashed line. 

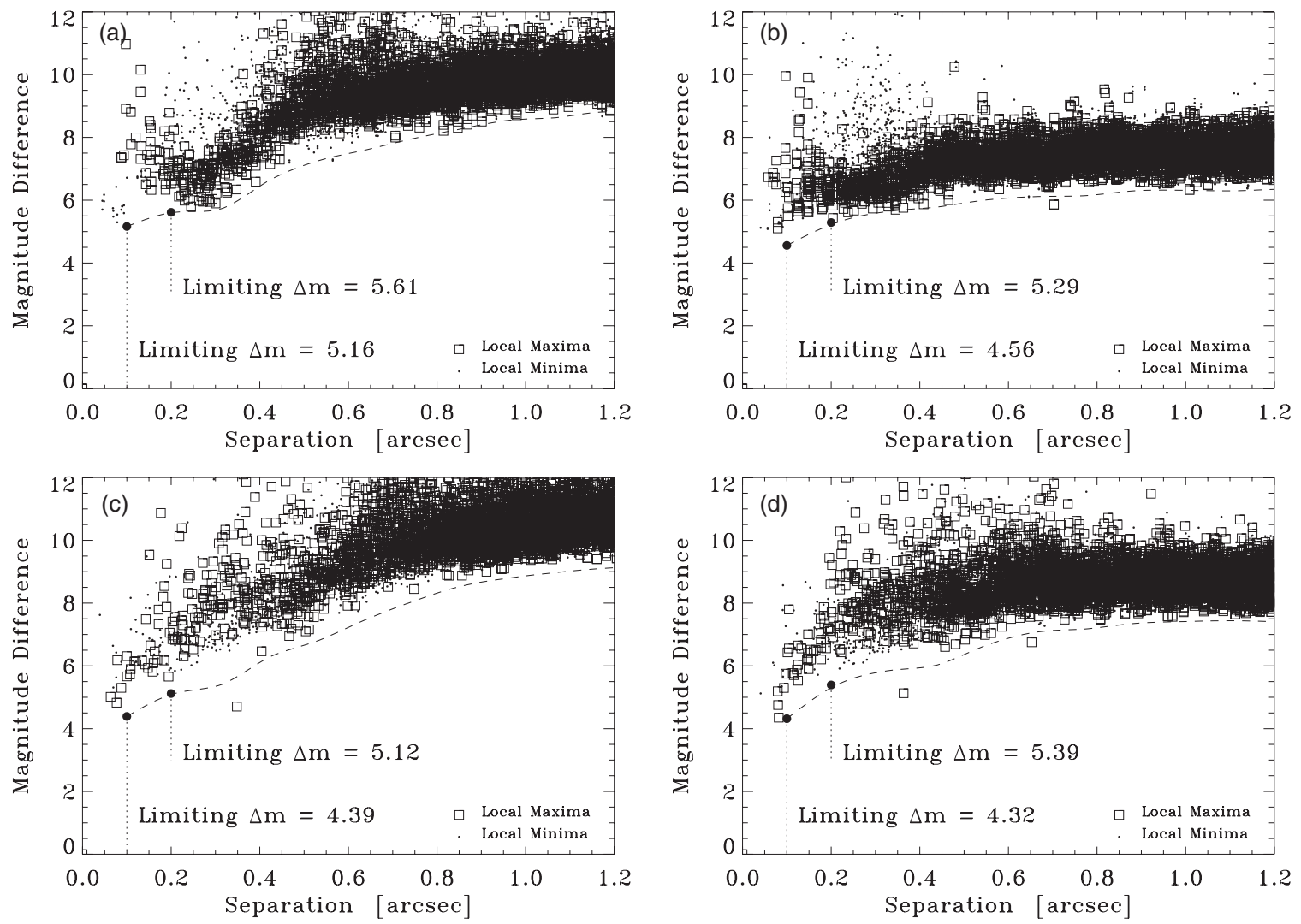

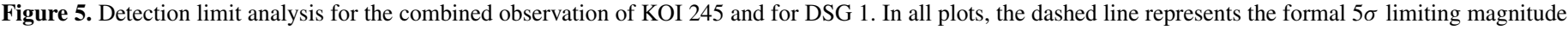

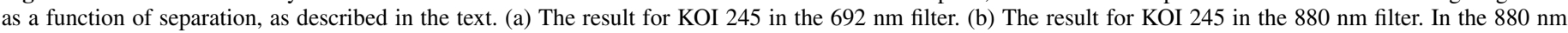

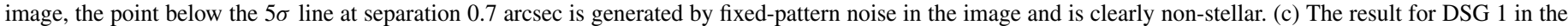

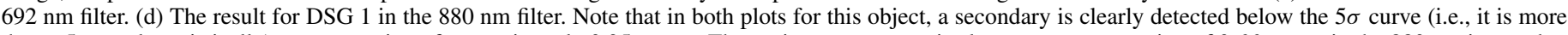

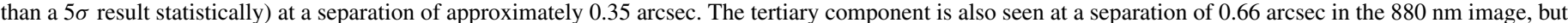
was not detected in the $692 \mathrm{~nm}$ image.

We stress that these limits are estimates that have some uncertainty. Particularly in the regime of faint objects (nominally $R>12$ at Gemini), the statistics of the reconstructed images become non-Gaussian. In the low signal-to-noise regime, two main sources of systematic noise begin to contribute to the distribution of low-level peaks (and valleys) in the reconstructed image. The first is a low-level line or cross pattern centered on the object. This appears to be related to the subtraction of the photon noise bias in the correlation functions that are used to make the reconstructed image. Some of the pixel values along the line or cross can be interpreted as a peak or valley with significance greater than $5 \sigma$. This is the cause of the one point in Figure 5(b) that are below the $5 \sigma$ line at a separation of approximately 0.7 arcsec. It is easy to rule these out as a stellar sources. The second type of pattern noise is in the form of lowlevel fringes that appear throughout the reconstructed image of low but varying intensity. The effect of this is to change the distribution of peaks from Gaussian or near-Gaussian (which is obtained in most high signal-to-noise reconstructed images) to a distribution that has a quasi-Gaussian core but broader wings. Therefore, it is not possible to associate the exact probability of detection one would naively assume from Gaussian statistics with the $5 \sigma$ lines drawn. We plan to study this effect in more detail so that more robust statements can be made in the future. At present, we note that both sources of fixed-pattern noise mentioned here lead to image features that are "peaks" in the sense that they are local maxima, but which differ in size and/or appearance from stellar sources. By accepting all local maxima (and minima), we have included in our distributions sources that clearly are not stellar. However, given the nature of the data, we judge this as the safest and best approach at this stage, even though it does make the interpretation of the $5 \sigma$ line more difficult.

The detection limits that we present here for the objects in Table 2 will provide one piece of ground-based evidence that should help further the analysis of these stars as hosts for planetary systems. For example, the planetary system of KOI 245 will be discussed in detail in a separate Kepler paper (Barclay et al. 2012). Two other objects, KOI 2115 and KOI 2044 , were in extremely crowded fields and are members of the open cluster NGC 6811; our non-detections could help establish that these objects do have planetary systems, which would be the first such detections for stars in the cluster environment.

In Figure 6, we plot the limiting magnitude difference as a function of object magnitude (Kepler magnitude, in most cases) for the non-detections in Table 2. This figure can be directly compared with Figure 8 in Howell et al. (2011), which shows the same result for WIYN data, and in fact we include in our figure here a shaded region that shows where the majority of points lie for WIYN from that figure. (The plot for WIYN data is very broad due to factors such as seeing variations and residual atmospheric dispersion.) This makes clear that the Gemini data are significantly better than WIYN data in the detection of faint companions. The mean trend of the Gemini data lies nearly a magnitude above the upper limit for typical WIYN data, and $\sim 2$ mag above the mean trend for WIYN, throughout most of the diagram. Gemini data are also of high quality to at least magnitude 16.5, meaning that a component as faint as 


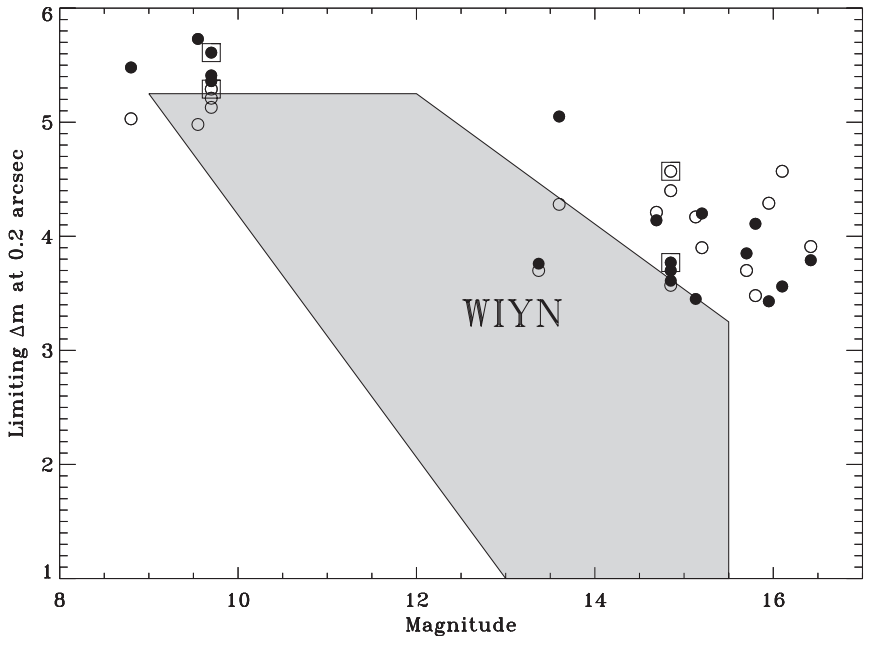

Figure 6. Limiting magnitude difference as a function of target magnitude for high-quality non-detections. Filled circles are the results at $692 \mathrm{~nm}$, and open circles are the results at $880 \mathrm{~nm}$. The boxed points are cases where the results on both nights were combined, as described in the text. The shaded area marks the region of most points in the same plot for WIYN data appearing in Howell et al. (2011). All points are marked at the estimated $5 \sigma$ detection limit at separation 0.2 arcsec; as discussed in the text, doubling the observation time would move points upward by $\sim 0.25 \mathrm{mag}$, and if $3 \sigma$ limits are instead desired, points would move upward by $0.55 \mathrm{mag}$.

magnitude 20.5 could be detected around such a primary star. While we have studied the behavior of the data at 0.2 arcsec in Figure 6, other separations may be of interest. In Figure 7, we show the degree to which larger separations result in a deeper limiting magnitude at two other separations, 0.6 and 1.0 arcsec. A significant improvement in the limiting magnitude is seen for brighter objects, but at fainter magnitudes, the improvement is more modest.

There are two cases in Table 2 where we observed the target on both July 27th and 28th, KOI 245 and KOI 2401. The first of these objects is on the brighter end of Kepler targets, $R \sim 9.7$, while the latter is on the fainter end, $R \sim 14.7$. For these objects, we have analyzed the data for each night separately, but we also combined the data, whereupon we find for example at 0.2 arcsec from the primary, the limiting $\Delta m$ is $0.26 \pm 0.11$ mag fainter than the average of the individual observations (the uncertainty here is the standard error of the four cases we have, two objects

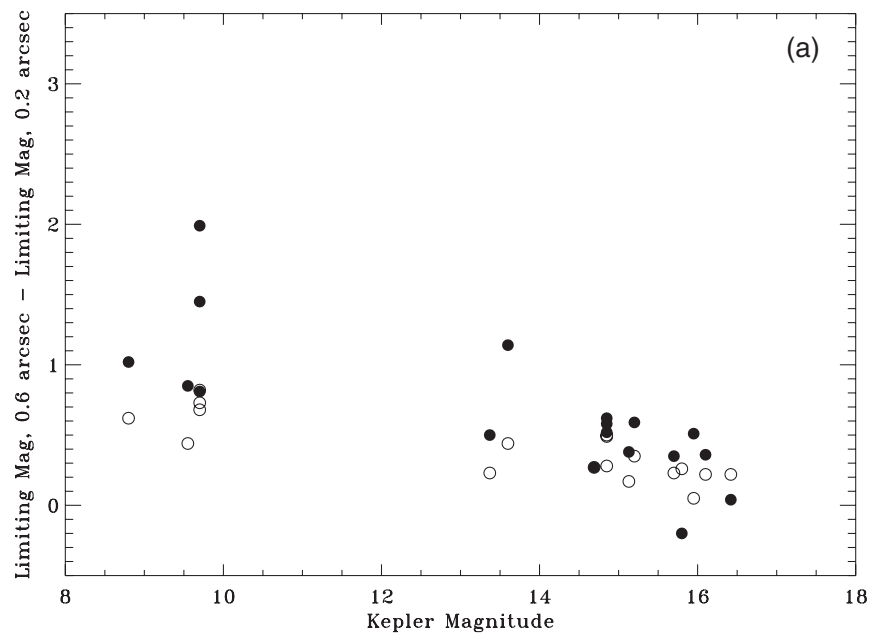

observed in two filters each). The combined observations are shown as boxed in Figure 6. Since in both cases the object was observed in the same way (i.e., the same number of frames on each night), we conclude that doubling the observation time leads to an extra quarter of a magnitude in the limiting $\Delta m$. Therefore, in Figure 6, points could be moved upward at the cost of more observing time; for example, in the brightest objects, where only 1000 frames were obtained leading to a limit of $\sim 5.5 \mathrm{mag}$, this could be pushed to at least 6.25 by observing the object for eight times as long, i.e., 8000 frames. The increase in $\Delta m$ of $0.25 \mathrm{mag}$ is slightly less than the value that would be expected from Gaussian statistics, which would be $2.5 \times \log (\sqrt{2})=0.38 \mathrm{mag}$. It may be that the two noise sources mentioned above are affecting this result at low signal to noise. We have also tried pairing different point-source observations with a given target to determine the effect of mismatch in seeing conditions and/or residual dispersion; cases where the point source appears well matched to the target produce limits that are about 0.2 mag fainter than poorer matches. This indicates that the point-source calibrator does limit these results at some level, and the point-source deconvolution may be another reason that the improvement is less than Gaussian.

Our detection limit of $5 \sigma$ is intentionally conservative; if $3 \sigma$ is instead desired, this would move points upward in Figure 6 by $0.55 \mathrm{mag}$, meaning that for, e.g., a 12th magnitude object, a reconstructed image with a $\Delta m$ limit of nearly 7 mag could be obtained with an 8000 -frame observation. It may also be possible to make further gains in the limiting $\Delta m$ by improving the reduction and analysis method. For example, we are currently studying a method for generating the pointsource data from a library of point sources taken at different sky positions. This may allow us to isolate and reduce the systematic component to the $\Delta m$ limits due to point-source mismatch.

\section{ANALYSIS AND DISCUSSION}

\subsection{Two Preliminary Orbit Determinations}

In the case of two systems, the data presented here together with data in the literature permit a first estimation of orbital elements. We caution that these are preliminary at this stage, but because of general consistency with all of the other information available, these orbits should be sufficiently useful over the next few years, until better orbits can be calculated. Table 4 shows

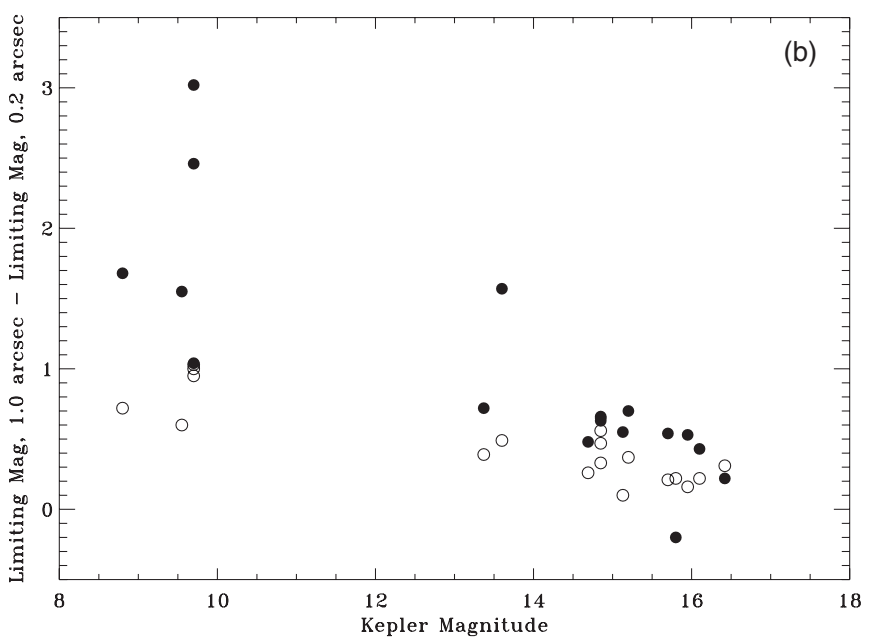

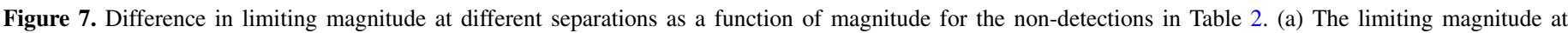

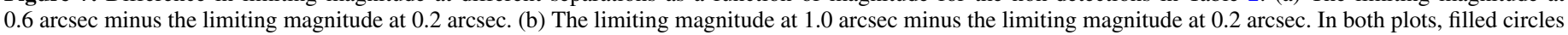
are observations taken in the $692 \mathrm{~nm}$ filter, and open circles are observations taken in the $880 \mathrm{~nm}$ filter. 
Table 4

Preliminary Orbits for Two Systems

\begin{tabular}{lcc}
\hline \hline Parameter & YSC 13 & YSC 134 \\
\hline$P$, years & $12.91 \pm 0.94$ & $0.4562 \pm 0.0013$ \\
$a$, mas & $99 \pm 10$ & $29.8 \pm 1.3$ \\
$i$, degrees & $52.0 \pm 6.6$ & $113.0 \pm 5.3$ \\
$\Omega$, degrees & $259 \pm 20$ & $31.3 \pm 2.6$ \\
$T_{0}$, years & $2003.36 \pm 0.44$ & $2010.167 \pm 0.014$ \\
$e$ & $0.708 \pm 0.084$ & $0.204 \pm 0.059$ \\
$\omega$, degrees & $70.5 \pm 8.7$ & $85.0 \pm 7.3$ \\
\hline
\end{tabular}

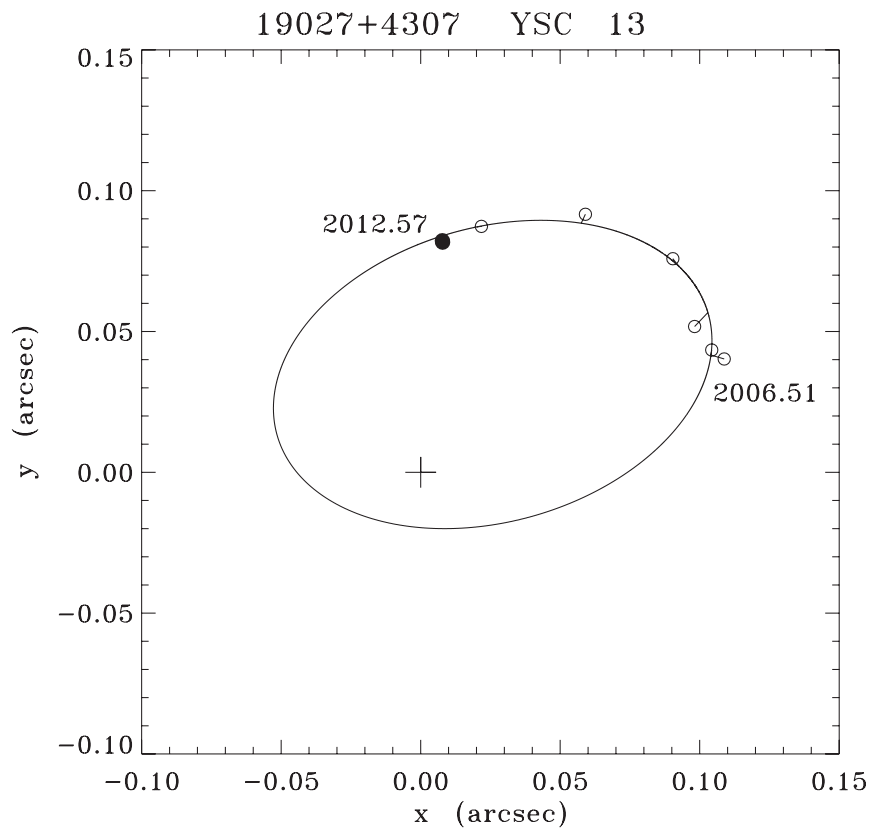

Figure 8. Orbit calculated here for YSC $13=$ HIP 93511 together with data from the literature and our measures from Table 1. The latter are shown with filled circles. All points are drawn with line segments from the data point to the location of the ephemeris prediction on the orbital path. North is down and east is to the right.

the orbital elements in both cases, which were obtained using the code described in MacKnight \& Horch (2004).

The first of these systems is YSC $13=$ HIP 93511. This system was listed as suspected double in the Hipparcos Catalogue (ESA 1997) and first resolved by us at the WIYN telescope in 2006 (reported in Horch et al. 2008). Since that time, we have observed the system several more times, and the data have shown a clear orbital trajectory. Currently, the separation is decreasing. The object is in the Kepler field, and T. Appourchaux (2012, private communication) has studied the photometric data from the satellite in order to determine masses of the two stars from pulsation frequencies. Classical orbital elements are of significant interest en route to dynamical masses to compare to the pulsation work. The Geneva-Copenhagen Spectroscopic Catalogue (Nordström et al. 2004) lists this object as slightly metal-poor with $[\mathrm{Fe} / \mathrm{H}]$ of -0.20 . The updated Hipparcos parallax (van Leeuwen 2007) is $9.98 \pm 0.47$ mas, and the spectral type is listed as F5 in the Hipparcos Catalogue.

Our orbit for the system gives a period of approximately 13 years, with the semi-major axis of 99 mas, so that the implied mass sum at this point is $5.8 \pm 2.1$ solar masses. The orbit is shown in Figure 8. Given that the magnitude difference of the pair appears to be around 1, we might guess that we have an F2 primary and an F8 secondary. On the solar-metallicity

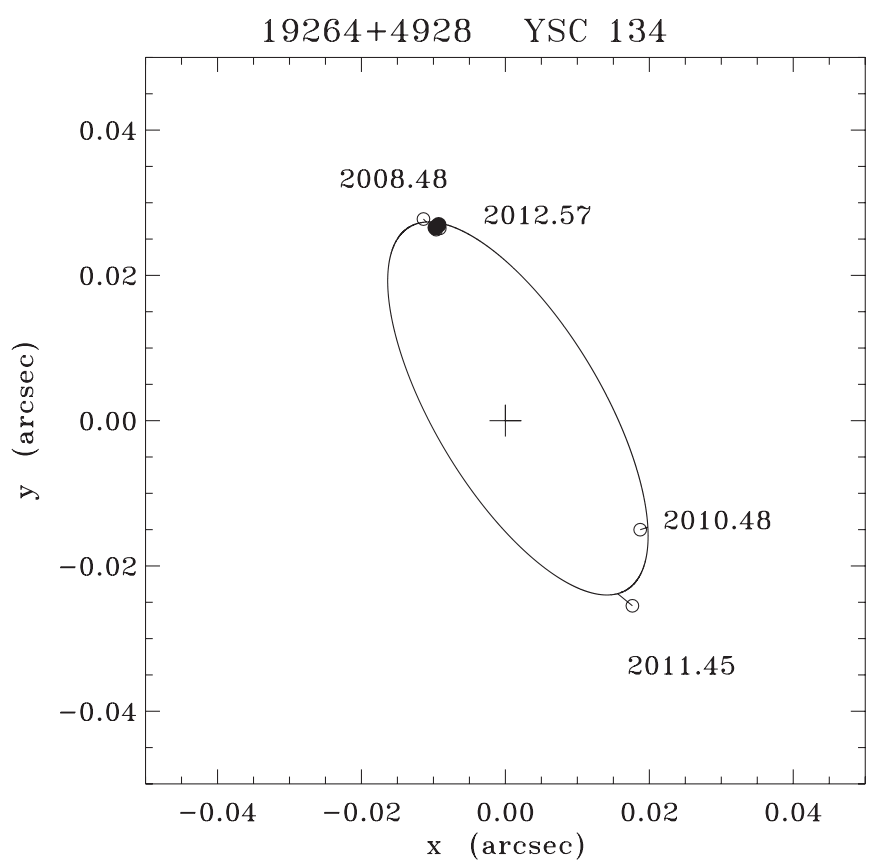

Figure 9. Orbit calculated here for YSC $134=$ HIP 95575 together with data from the literature and our measures from Table 1. The latter are shown with filled circles. All points are drawn with line segments from the data point to the location of the ephemeris prediction on the orbital path. North is down and east is to the right.

main sequence this would imply a mass sum of $\sim 3$ solar masses (using information in Schmidt-Kaler 1982), meaning the orbital mass sum is marginally consistent with this, given the large uncertainty. Hopefully, the coming years will yield more astrometry of the system and the dynamical mass can then be compared in a more meaningful way with the final results from Kepler. We encourage other observers to contribute measures in the coming years for this reason.

The second system for which we computed an orbit is YSC $134=$ HIP 95575. A spectroscopic orbit exists due to Tokovinin (1991), and the object is listed as a metal-poor double-lined spectroscopic binary in the Geneva-Copenhagen Catalogue with $[\mathrm{Fe} / \mathrm{H}]$ of -0.80 and mass ratio of $0.934 \pm 0.022$. It is also known to be binary due to the work of Jancart et al. (2005), where an astrometric orbit is deduced from Hipparcos data. Both Balega et al. (1991) and Mason et al. (2001a; B. D. Mason et al. 2012, in preparation) have observed this pair without resolving the stars; our first measurement of the separation at WIYN was in 2008 (reported in Paper III). Since that time, we have successfully resolved the system in 2010 and 2011 at WIYN, and can now add the Gemini data to the orbit calculation. As there are still very few points with which to work, we searched for an orbital period of approximately 0.5 years, i.e., close to that of the known astrometric and spectroscopic orbits, though we did not constrain our fit to either period. (Since there are few data points, some multiples of this period can also be made to fit the data with comparable reduced- $\chi^{2}$.) Nonetheless, as the final orbit shows in Figure 9, there is a very good match to the data with our final parameters, including our derived period of $0.4562 \pm 0.0013$ years. This matches both the Jancart and Tokovinin periods within the uncertainty, and our other orbital elements are in reasonable agreement with the Tokovinin solution in cases where a comparison can be made.

The spectral type of YSC 134 is listed as $\mathrm{K} 3 \mathrm{~V}$ in the Hipparcos Catalogue, and the (updated) parallax found there is 
$39.98 \pm 0.73$ mas. Combining this with our orbital parameters, a mass sum of $2.0 \pm 0.3$ solar masses is obtained. The magnitude difference is modest, about 0.8 , so that if this has a composite spectral type of $\mathrm{K} 3 \mathrm{~V}$, it would be roughly a $\mathrm{K} 1 \mathrm{~V}+\mathrm{K} 4 \mathrm{~V}$ system, and one would expect a mass sum perhaps around 1.2-1.5 solar masses, accounting for the lower metallicity. This is lower than the value derived from the orbit here, but at this early stage the dynamical result is still not unreasonable, and the orbital elements should be serviceable for the time being. The above assignment of spectral types for the two stars also yields a mass ratio of $\sim 0.9$, in good agreement with the value from the Geneva-Copenhagen Catalog. Finally, we note that this picture is also consistent with the non-detections of Balega et al. and Mason et al.-in all three of those cases, the separation was below the detection limit as stated by those observers at the time of observation, based on our orbital parameters.

\subsection{Comments on Other Systems}

For the discussion below, parallax values mentioned are from van Leeuwen (2007), spectral types are those appearing in the Hipparcos Catalogue (ESA 1997), and mass ratios and [Fe/H] values are taken from the Geneva-Copenhagen Spectroscopic Catalogue (Nordström et al. 2004).

\subsubsection{YSC 135}

From the data available, this system may have a period of less than one year; it has $[\mathrm{Fe} / \mathrm{H}]$ of -0.28 , spectral type $\mathrm{G} 5$, and parallax of $31.29 \pm 0.47$ mas. A mass ratio of 0.686 appears in the Geneva-Copenhagen Catalogue, but without uncertainty. Nonetheless, because of the relatively small parallax uncertainty, this system could contribute to our knowledge of the mass-luminosity relation relatively quickly.

\subsubsection{YSC 136}

Like YSC 135, this is a slightly sub-solar-metallicity pair $([\mathrm{Fe} / \mathrm{H}]=-0.11)$, and has mass ratio $0.754 \pm 0.013$, according to the Geneva-Copenhagen Catalogue. There are too few observations available at present to attempt an orbit, but given the orbital motion so far, this should be possible within the next couple of years, if further relative astrometry is obtained. The spectral type is G0.

\subsection{3. $\mathrm{COU} 2431 \mathrm{Aa}$}

B. D. Mason et al. (2012, in preparation) attempted to resolve this system recently but without success; we believe that our measure in Table 1 is the first resolution of this object, which has been known as a spectroscopic binary since the 1980s. There seem to be differing values of metallicity for this object in the literature, from as low as the value of Latham et al. (1988), $[\mathrm{Fe} / \mathrm{H}]=-0.78$, to the Geneva-Copenhagen Catalogue value of $[\mathrm{Fe} / \mathrm{H}]=-0.14$. The spectroscopic orbit of Goldberg et al. (2002) has a period of only 112 days and the parallax uncertainty from Hipparcos is $\sim 2 \%$, so further astrometric data on the system would allow for an excellent test of the metal-poor mass-luminosity relation, if indeed the system is significantly metal-poor.

\subsubsection{WSI $6 A B$}

Our observations here confirm that the known double WSI 6 is comprised of at least one additional star. This was expected since the object also has a spectroscopic orbit with period 378 days due to Latham et al. (1992), and the orbital data of WSI 6AB have made clear that it is not the spectroscopic component. This system is also reportedly metal-poor, with Latham et al. having estimated $[\mathrm{Fe} / \mathrm{H}]$ at -1.01 . WSI $6 \mathrm{AB}$ probably has an orbital period on the order of 20 years, and Jancart et al. (2005) confirm the spectroscopic period with their astrometric orbit of the inner pair. Thus, the data are beginning to accumulate to the point where a triple fit could soon be attempted. The key element of this would be more resolved observations of the Aa component.

\section{CONCLUSIONS}

We have presented results of 71 speckle observations of binary and unresolved stars. In 55 cases, position angles, separations, and/or magnitude differences are presented, and for the remainder, detection limits for faint companions are given. These observations outline the potential for DSSI speckle observing at the Gemini North Telescope, for projects such as high-resolution imaging of Kepler targets thought to harbor Earth-like planets, as well as precision astrophysics of binary stars, resolvable sources, crowded field sources, and faint targets.

We thank the Kepler Science Office located at the NASA Ames Research Center for providing partial financial support for the upgraded DSSI instrument. It is also a pleasure to thank Steve Hardash, Andy Adamson, Inger Jorgensen, and the entire summit crew for their excellent work in getting the instrument to the telescope and installing it. We appreciate the collaboration of Thierry Appourchaux in pointing out the potential importance of YSC 13 in the determination of stellar masses from Kepler data. This work was funded by the Kepler Science Office and NSF Grant AST-0908125. It made use of the Washington Double Star Catalog maintained at the U.S. Naval Observatory and the SIMBAD database, operated at CDS, Strasbourg, France.

\section{REFERENCES}

Balega, I. I., Balega, Y. Y., Vasyuk, V. A., \& Tokovinin, A. A. 1991, SvAL, 17, 530

Barclay, T., Rowe, J. F., Lissauer, J. J., et al. 2012, Nature, submitted

ESA 1997, The Hipparcos and Tycho Catalogues (ESA SP-1200; Noordwijk: ESA)

Goldberg, D., Mazeh, T., Latham, D. W., et al. 2002, AJ, 124, 1132

Hartkopf, W. I., Mason, B. D., \& Worley, C. E. 2001a, AJ, 122, 3472

Hartkopf, W. I., McAlister, H. A., \& Mason, B. D. 2001b, AJ, 122, 3480

Horch, E. P., Franz, O. G., Wasserman, L. H., \& Heasley, J. N. 2006, AJ, 132,836

Horch, E. P., Gomez, S. C., Sherry, W. H., et al. 2011a, AJ, 141, 45 (Paper II)

Horch, E. P., van Altena, W. F., Cyr, W. M., Jr., et al. 2008, AJ, 136, 312

Horch, E. P., van Altena, W. F., Howell, S. B., Sherry, W. H., \& Ciardi, D. R. 2011b, AJ, 141, 180 (Paper III)

Horch, E. P., Veillette, D. R., Baena Gallé, R., et al. 2009, AJ, 137, 5057 (Paper I)

Howell, S. B., Everett, M. E., Horch, E. P., \& Ciardi, D. R. 2012, PASP

Howell, S. B., Everett, M. E., Sherry, W. H., Horch, E. P., \& Ciardi, D. R. 2011, AJ, 142, 19

Jancart, S., Jorrisen, A., Babusiaux, C., \& Pourbaix, D. 2005, A\&A, 442, 365

Latham, D. W., Mazeh, T., Carney, B. W., et al. 1988, AJ, 96, 567

Latham, D. W., Mazeh, T., Stefanik, R. P., et al. 1992, AJ, 104, 774

Lohmann, A. W., Weigelt, G., \& Wirnitzer, B. 1983, ApOpt, 22, 4028

MacKnight, M., \& Horch, E. P. 2004, BAAS, 36, 788

Mason, B. D., Hartkopf, W. I., Holdenried, E. R., \& Rafferty, T. J. 2001a, AJ, 121,3224

Mason, B. D., Wycoff, G. L., Hartkopf, W. I., Douglass, G. G., \& Worley, C. E. 2001b, AJ, 122, 3466

Meng, J., Aitken, G. J. M., Hege, E. K., \& Morgan, J. S. 1990, JOSAA, 7, 1243

Nordström, B., Mayor, M., Andersen, J., et al. 2004, A\&A, 419, 989

Schmidt-Kaler, T. 1982, in Landolt-Börnstein New Series, Group 6, Vol. 2b, Stars and Star Clusters, ed. K. Schaefers \& H.-H. Voigt (Berlin: Springer), 1 Tokovinin, A. A. 1991, A\&AS, 91, 497

van Leeuwen, F. 2007, A\&A, 474, 653 